\title{
Trajectories of brain aging in middle-aged and older adults: Regional and individual differences
}

\author{
Naftali Raz ${ }^{\text {a,b,* }}$, Paolo Ghisletta ${ }^{c}$, Karen M. Rodrigue ${ }^{d}$, Kristen M. Kennedy ${ }^{\text {d }}$, Ulman Lindenberger ${ }^{\mathrm{e}}$ \\ a Institute of Gerontology, Wayne State University, USA \\ b Department of Psychology, Wayne State University, USA \\ c Faculty of Psychology and Educational Sciences, University of Geneva, Switzerland Distance Learning University, Sierre, Switzerland \\ d School of Behavioral and Brain Sciences, University of Texas at Dallas, Dallas, TX, USA \\ e Max Planck Institute for Human Development, Berlin, Germany
}

\section{A R T I C L E I N F O}

\section{Article history:}

Received 17 December 2009

Revised 3 March 2010

Accepted 8 March 2010

Available online 16 March 2010

\section{Keywords:}

Aging

Longitudinal

MRI

Brain

Volume

Hypertension

ApoE

Sex differences

\begin{abstract}
A B S T R A C T
The human brain changes with age. However, the rate and the trajectories of change vary among the brain regions and among individuals, and the reasons for these differences are unclear. In a sample of healthy middle-aged and older adults, we examined mean volume change and individual differences in the rate of change in 12 regional brain volumes over approximately 30 months. In addition to the baseline assessment, there were two follow-ups, 15 months apart. We observed significant average shrinkage of the hippocampus, entorhinal cortex, orbital-frontal cortex, and cerebellum in each of the intervals. Shrinkage of the hippocampus accelerated with time, whereas shrinkage of the caudate nucleus, prefrontal subcortical white matter, and corpus callosum emerged only at the second follow-up. Throughout both assessment intervals, the mean volumes of the lateral prefrontal and primary visual cortices, putamen, and pons did not change. Significant individual differences in shrinkage rates were observed in the lateral prefrontal cortex, the cerebellum, and all the white matter regions throughout the study, whereas additional regions (medialtemporal structures, the insula, and the basal ganglia) showed significant individual variation in change during the second follow-up. No individual variability was noted in the change of orbital frontal and visual cortices. In two white matter regions, we were able to identify factors associated with individual differences in brain shrinkage. In corpus callosum, shrinkage rate was greater in persons with hypertension, and in the pons, women and carriers of the ApoE\&4 allele exhibited declines not noted in the whole sample.
\end{abstract}

(c) 2010 Elsevier Inc. All rights reserved.
The extant literature on human brain aging converges onto a pessimistic conclusion: the brain deteriorates with age. According to multiple cross-sectional studies of healthy adults, advanced age is associated with smaller brains and thinner cortices (e.g., Raz et al., 1997; Jernigan et al., 2001; Raz et al., 2004; Fjell et al, 2009a; see Raz and Kennedy (2009) for a review of the findings). Longitudinal investigations show that in healthy adults, brain parenchyma shrinks within a span of several years (e.g., Pfefferbaum et al., 1998; Resnick et al., 2003; Scahill et al., 2003; Raz et al., 2005; Driscoll et al., 2009; Raz and Kennedy, 2009), and, according to a recent report even in shorter periods of time (Fjell et al., 2009b). However, structural brain changes are regional and differential. Whereas the tertiary association cortices, the neostriatum, and the cerebellum show the greatest rate of shrinkage, the primary visual cortices evidence much smaller changes (Raz et al., 2005; Driscoll et al., 2009; Fjell et al., 2009b). Moreover, the regional rates of age-related declines may depend on common age-related vascular risk factors (Resnick et al., 2003; Raz

\footnotetext{
* Corresponding author. 87 East Ferry St., Detroit, MI 48202, USA. Fax: + 1508256 5689.

E-mail address: nraz@wayne.edu (N. Raz).
}

et al., 2007, 2008) and genetic variants such as $\varepsilon 4$ allele of the apolipoprotein E (ApoE) gene (Moffat et al., 2000).

Scarcity of longitudinal studies hampers understanding the nature and mechanisms of age-related changes in brain structure. Furthermore, presumably because many studies aim at predicting the onset of dementia, they focus on older adults and individuals selected for elevated risk for dementia. Therefore, volumes of medial temporal lobe structures and global indices of brain health, such as ventricular size most frequently serve as the indices of brain aging, whereas other brain regions do not receive sufficient attention (e.g., Barnes et al., 2009; Stoub et al., 2008; see Raz et al. (2005) and Raz and Kennedy (2009) for reviews).

Cross-sectional design, which is still the most popular approach to brain aging research, is inherently incapable of measuring true change and gauging individual differences in brain shrinkage rates, not to mention the shape of trajectories that characterize brain aging. Some cross-sectional studies suggested that in selected regions (e.g., hippocampus, cerebellum, or prefrontal white matter) decline might accelerate with age (Jernigan et al., 2001; Raz et al., 2004) and longitudinal reports support that hypothesis at least regarding the hippocampus (Scahill et al., 2003; Raz et al., 2005; Fjell et al., 2009b) 
and the cerebellum (Raz et al., 2005). However, the vast majority of the extant longitudinal studies consist of only two waves of measurement, and the studies with more than two repeated assessments do not report tests of nonlinearity for the trajectories of change (Resnick et al., 2003; Rusinek et al., 2003, but see Fjell et al. (2009b)). Two multi-wave studies that explicitly examined nonlinearity of brain shrinkage reported multiple instances of age-related acceleration in decline of local volume, especially in the prefrontal (Fjell et al., 2009b; Driscoll et al., 2009) and superior parietal cortices (Driscoll et al., 2009), and the medial-temporal regions (Fjell et al., 2009b). Notably, the extant longitudinal studies report almost exclusively mean change in regional cerebral volumes and provide no estimates of individual differences in the rate of change. Thus, the extent of individual variability in regional brain shrinkage is largely unknown. It is, therefore, important to estimate the magnitude of individual differences in regional brain shrinkage and to determine whether the aging brain evidences a steady linear decline or shows significant acceleration of change and whether common risk factors affect the rate and the shape of decline trajectories.

Although statistical methods for evaluating change in the context of individual differences such as latent difference score models (McArdle, 2009; McArdle and Nesselroade, 2003) are widely available, only a few studies of brain aging have benefited from them (McArdle et al., 2004; Raz et al., 2005, 2008). The first was restricted to one brain measure, ventricular size obtained from computerized tomography (McArdle et al., 2004). The second and third (Raz et al., 2005, 2008), MRI studies of multiple brain regions, were limited to two measurement occasions.

In this study, our goal was to address the problematic aspects of previous investigations outlined above. The first aim was to evaluate the mean change of the regional brain volumes and individual variability of regional change over a relatively short time span. The second aim was to examine the shape of the trajectories of change and regional differences therein. Finally, we assessed the effect of common risk factors, hypertension and ApoE\&4 allele as well as sex and education, on the trajectories of regional brain aging.

\section{Method}

\section{Participants}

Healthy volunteers from the metropolitan Detroit area who responded to media advertisements and flyers participated in this study. All responders completed a health questionnaire and underwent telephone and personal interviews to screen for multiple health problems and counter-indicators to magnetic resonance imaging (MRI). Persons who reported a history of cardiovascular (except controlled and uncomplicated essential hypertension), neurological or psychiatric conditions, head trauma with loss of consciousness for more than $5 \mathrm{~min}$, thyroid problems, diabetes mellitus, and/or drug and alcohol problems were excluded from participation in the study. Individuals with metal implants and pacemakers and those who were taking anti-seizure medication, anxiolytics, or antidepressants did not participate in the study. All participants attained a minimum of high school education, were native English speakers, and were consistent right-handers (75\% on the Edinburgh Handedness Questionnaire; Oldfield, 1971). Of 673 persons who responded to advertising, 166 (less than 25\%) satisfied the screening criteria. Of those, 76 participants age 49 and older were eligible for the longitudinal study, and 40 agreed to participate. The majority of the participants (80\%) were Caucasians and 20\% were African-Americans.

Participants were screened for near, far, and color vision abnormalities (Optec 2000 vision tester; Stereo Optical Co., Inc., Chicago, IL) and deficits in speech-range hearing acuity (model MA27; Maico Diagnostics, Eden Prairie, MN). To exclude probable cases of dementia and depression, we used the Mini-Mental Status Examination (MMSE; Folstein et al., 1975) with a cut-off of 26, and Geriatric Depression Questionnaire (CES-D; Radloff, 1977) with a cut-off of 16. Only two men, one of them hypertensive, scored 26 on MMSE, and nobody scored above 12 on CES-D. All participants provided written informed consent and received debriefing in accord with university human investigations committee guidelines.

A trained technician measured systolic and diastolic blood pressure with an analog mercury sphygmomanometer (Model 12-525; Country Technology, Gays Mills, WI) equipped with a standard brachial cuff (Omron Professional) from participants who sat in a comfortable chair in a climate-controlled office. The measures were obtained once from each arm and averaged within each session. A different technician measured blood pressure on three different days. Thus, the blood pressure readings in this study constituted an average of six measures. To classify participants as hypertensives, we used, in addition to the fact of carrying a diagnosis and taking anti-hypertensive medications, an operational definition of systolic blood pressure greater than $140 \mathrm{~mm} \mathrm{Hg}$ and diastolic pressure greater than $90 \mathrm{~mm} \mathrm{Hg}$ (Joint National Committee on Prevention, Detection, Evaluation, and Treatment of High Blood Pressure, 1997).

Forty participants were examined at baseline, 37 returned for the first follow-up, and 30 returned for the second follow-up assessment. The average interval between baseline and the first follow-up was 15.93 ( \pm 1.85$)$ months, while between the two follow-ups it was $15.45( \pm 2.76)$ months. The dropouts did not differ from the persons who remained in the study in their mean age, blood pressure, and MMSE scores, but we noted a trend for greater attrition of less educated participants: $\chi^{2}(2)=5.06, p=0.08$.

\section{Genotyping for ApoE}

DNA was isolated from buccal cultures obtained in mouthwash samples using a Gentra Autopure LS under the standard buccal cell protocol (see Raz et al. (2009) for details). ApoE genotyping yielded four ApoEs2/3, $22 \varepsilon 3 / \varepsilon 3$, nine $\varepsilon 3 / \varepsilon 4$, one $\varepsilon 4 / \varepsilon 4$, and no $\varepsilon 2 / \varepsilon 2$ genotypes. Genotype on one participant was unavailable. Because of small frequency of some ApoE alleles and genotypes in this sample, we compared only 10 ApoE 44 carriers with 26 persons who had no $\varepsilon 4$ allele. Frequency of ApoEs4 did not differ between Caucasian and African-American participants: $\chi^{2}=1.99, p=16$.

\section{MRI protocol}

MR images were acquired on the same 1.5 Tesla Siemens Magnetom Sonata scanner (Siemens Medical Systems, Erlangen, Germany) for all three waves. The regional volume measures were obtained from a 3D high resolution magnetization-prepared rapid gradient-echo (MPRAGE) sequence acquired in the coronal plane with echo time $(\mathrm{TE})=3.93 \mathrm{~ms}$, repetition time $(\mathrm{TR})=800 \mathrm{~ms}$, inversion time $(\mathrm{TI})=420 \mathrm{~ms}$, field of view $(F O V)=192 \times 192 \mathrm{~mm}$, acquisition matrix $=256 \times 256 \mathrm{~mm}$, flip angle $=20^{\circ}$, and voxel size $=0.75 \times 0.75 \times 1.5 \mathrm{~mm}^{3}$. Acquisition of 144 slices took $7.41 \mathrm{~min}$.

\section{Image processing and manual morphometry}

Image processing and regional volume measures are described in detail elsewhere (Raz et al., 2004, 2005). The images acquired on all three occasions were coded, and a person other than the tracers randomized the order of their tracing within each participant. The tracers were blind to the time of acquisition of the specific images and to the demographic characteristics of the participants. Reliability of all ROI measures (intraclass correlation for random raters) (Shrout and Fleiss, 1979) exceeded ICC2 $>0.93$.

The volumes were computed from measured areas of the ROIs (see Raz et al. (2003a,b, 2004) for details). In addition to the intracranial vault (ICV), the following 12 ROIs were measured: lateral prefrontal cortex (LPFC), orbital frontal cortex (OFC), the adjacent prefrontal white matter (PFw), primary visual (calcarine) cortex (VC), the hippocampus 
(HC), the entorhinal cortex (EC), the insula (In), the neostriatal nuclei (caudate, Cd and Putamen, Pt), the corpus callosum (CC), the ventral pons, and the cerebellar hemispheres $(\mathrm{CbH})$. The illustrations of the traced ROIs are presented in Figs. 1 and 2.

Demarcation and tracing of region of interest (ROIs)

Intracranial vault (ICV)

The ICV was estimated from the coronal sections. It covered all contents within the cranial cavity, including the brain stem and cerebellum. The operator traced ICV on every eight slices (a total of 11-12 slices; $12 \mathrm{~mm}$ interslice distance) between the first slice following the orbits and last slice on which brain tissue was visible. Previous work indicates that there is minimal reduction in accuracy and reliability at this sampling density compared to tracing every slice (Eritaia et al., 2000). The reliability of this measure was ICC(2) $=0.99$.

\section{Lateral prefrontal cortex (LPFC)}

Lateral prefrontal cortex was measured on the 8-12 coronal slices located within the posterior $40 \%$ of the distance between the genu of the corpus callosum and the frontal pole. The reliability of this measure was $\operatorname{ICC}(2)=0.97$.

Orbito-frontal cortex (OFC) was measured on the same coronal slices as the lateral prefrontal. The reliability of that measure was ICC $(2)=0.95$.

Primary visual (calcarine) cortex (VC)

The region was defined as the cortical ribbon lining the calcarine sulcus. The inferior and superior boundaries of this ROI were demarcated by the point of sulcus opening. At this point a line was drawn horizontally so that no cortex (dorsal or ventral) outside of the calcarine sulcus was included, confining this ROI to a part of area 17, and excluding other areas. The reliability of this measure was $\operatorname{ICC}(2)=0.94$.

Insula (In)

The rules for this ROI have not been previously reported and are presented here in detail. The insula was traced on the coronal plane on approximately 22 slices beginning at the optic chiasm (the first slice on which the optic tracts become continuous) continuing to the posterior commissure. The insular cortex lies inside the lateral fissure, and is demarcated dorsally and ventrally by the circular sulcus. The medial boundary is the white matter lateral to the claustrum, whereas the lateral boundary is the gray matter of the insular cortex. Tracing begins at the dorsal medial edge of the circular sulcus and continues medially along the border of the white matter, returning along the gray matter. The reliability of this measure was $\operatorname{ICC}(2)=0.96$.

\section{Hippocampus (HC)}

Hippocampal volume was measured on continuous slices aligned perpendicular to the long axis of the hippocampus between the mammillary bodies and the slice showing the fornices rising from the fimbria. The hippocampus included sectors CA1-CA4, the dentate gyrus and the subiculum. The reliability of this measure was ICC $(2)=$ 0.95 .

\section{Entorhinal cortex (EC)}

In tracing this region, we followed the rules developed by Insausti et al. (1998) with minor modifications. The only change in our rules

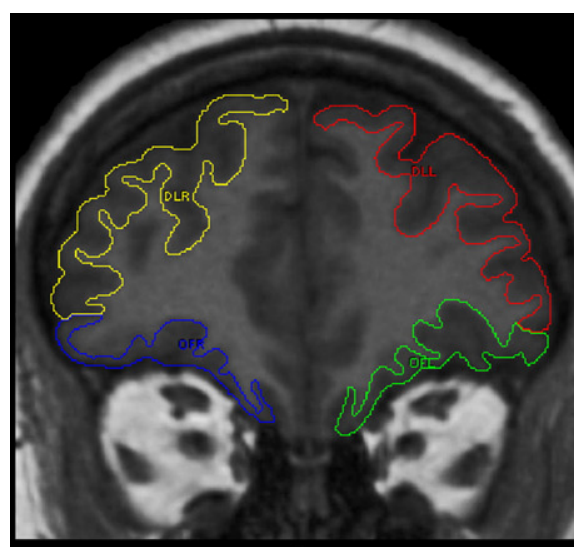

A

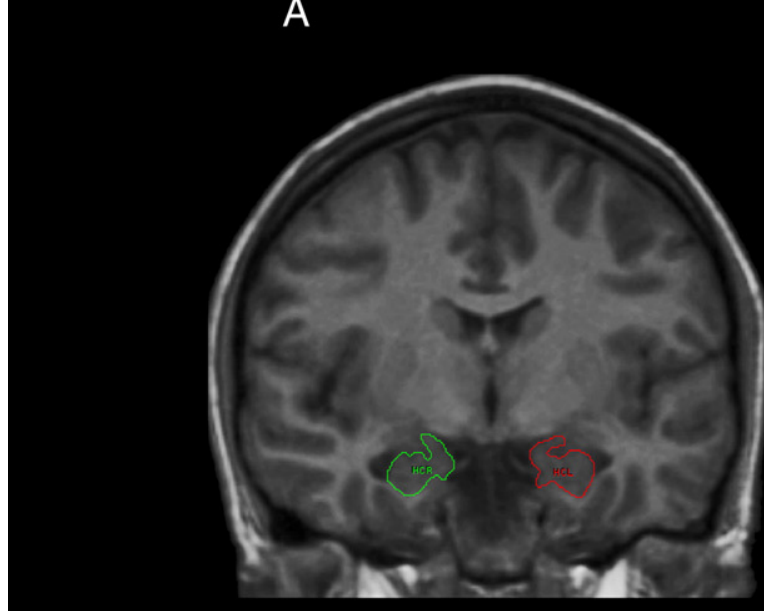

D

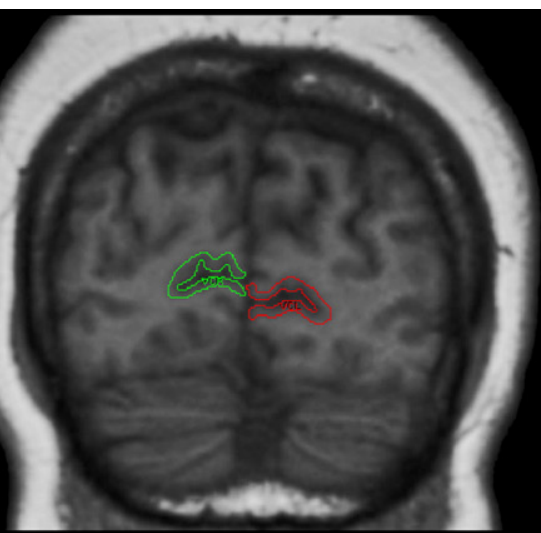

B
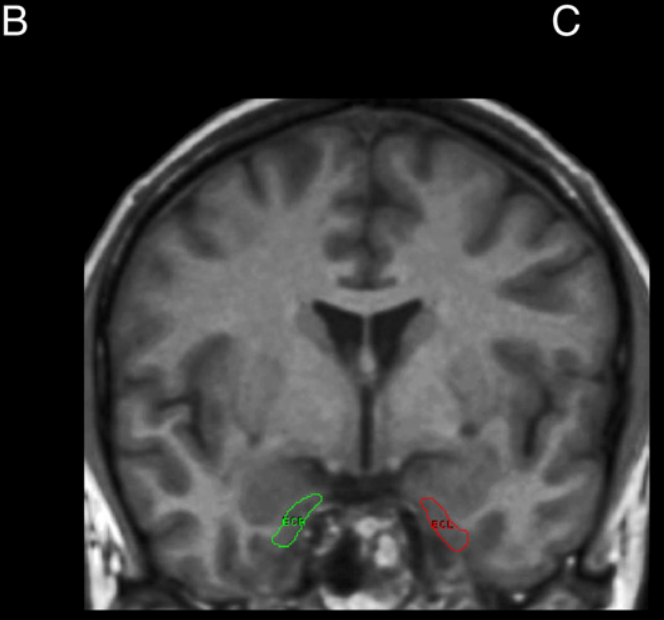

E

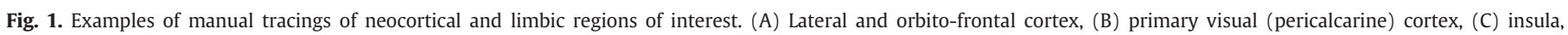
(D) hippocampus, and (E) entorhinal cortex. 


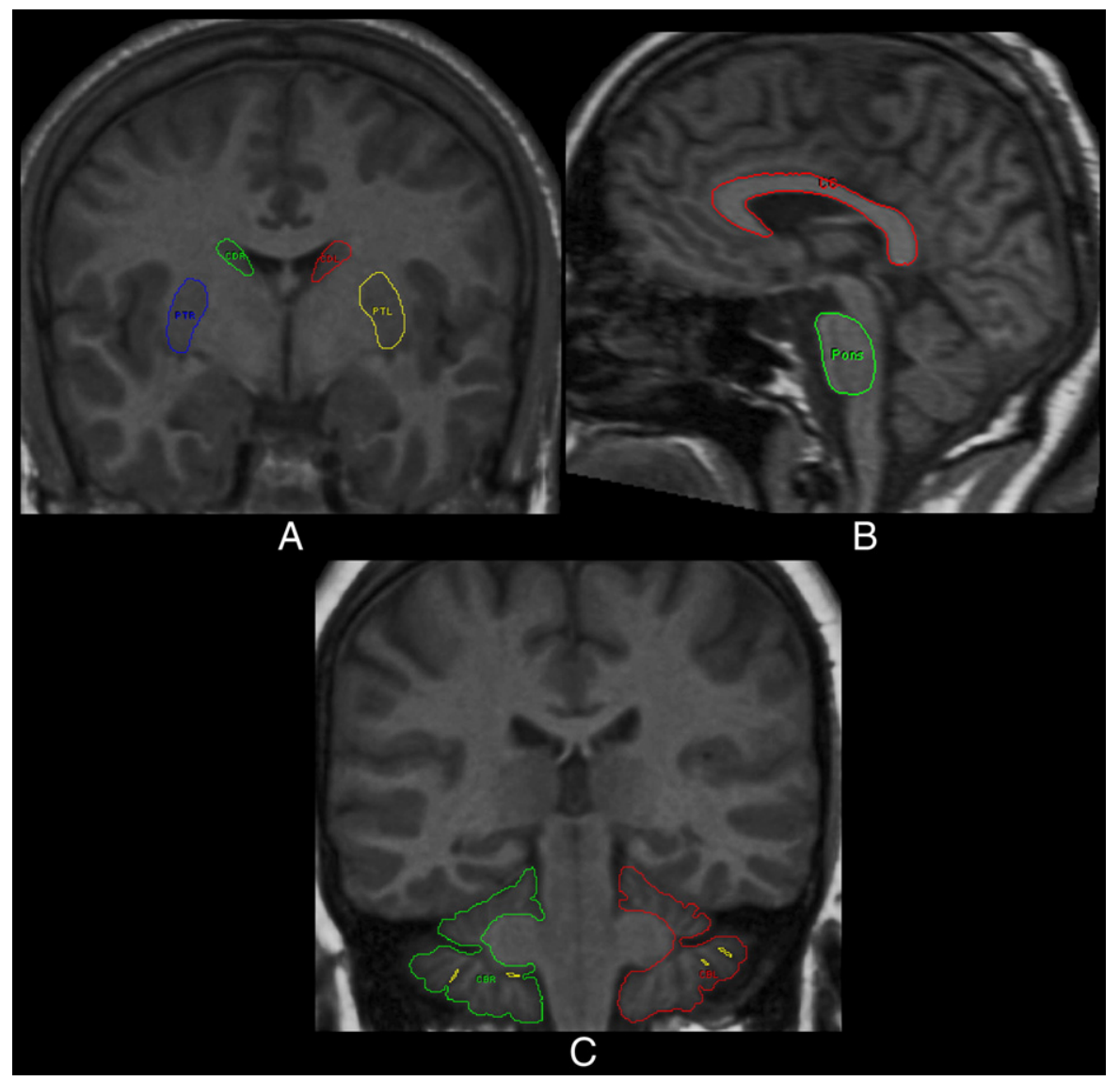

Fig. 2. Examples of manual tracings of the caudate nucleus and putamen (A), corpus callosum and pons (B), and cerebellar hemispheres (C).

was the specific point of the collateral sulcus - the medial bank - that was used to determine the ventro-lateral boundary. The modification is conservative and helps to avoid overestimation of the EC volume. The EC areas were measured on 12-17 slices per subject. The reliability of this measure was $\operatorname{ICC}(2)=0.93$.

\section{Prefrontal white matter (PFw)}

The range is identical to lateral prefrontal and orbital frontal cortices and includes subcortical white matter associated with both cortical regions, but excludes the ventricles and other cerebrospinal fluid spaces. The reliability of this measure was $\operatorname{ICC}(2)=0.95$.

\section{Caudate nucleus (Cd)}

The volume of the head and the body of the caudate was estimated from 15 to 20 coronal slices. The most rostral slice was the one on which the caudate first appeared; usually lateral to the lateral ventricles. On the one to three sections on which the nucleus accumbens was visible, a diagonal line was drawn from the most inferior tip of the internal capsule to the lateral ventricle. The caudate was traced on every other slice (interslice distance $3 \mathrm{~mm}$ ) until no longer visible. The reliability of this measure was $\operatorname{ICC}(2)=0.95$.

\section{Putamen (Pt)}

The volume of the putamen was measured from 15 to 20 coronal slices (every other slice at the interslice distance of $3 \mathrm{~mm}$ ). The operator began tracing on the most rostral slice on which the putamen was visible and continued to the most caudal slice on which it could be detected. The external capsule demarcated the putamen laterally throughout the ROI. The dorsal boundary of the putamen was the white matter. The internal capsule delineated the medial border until reaching the anterior commissure. Past that point, the globus pallidus became the medial border of the putamen. The limen insulae, optic radiations, the amygdala, the temporal horn, and the anterior commissure served as the ventral boundary of the putamen on various slices. The reliability of this measure was $\operatorname{ICC}(2)=0.93$.

\section{Cerebellar hemispheres $(\mathrm{CbH})$}

The cerebellar hemispheres were traced on every coronal slice on which they were visible, including the hemispheric gray and white matter, corpus medullare, and cerebellar tonsils. The vermis, the superior medullary velum, and the fourth ventricle were excluded. The cerebellar peduncles were included in the cerebellar white matter on the slices that preceded the appearance of the anterior vermis because they were difficult to distinguish from the surrounding white matter. Caudally to that slice, the peduncles were easily distinguishable from the cerebellar gray matter and were reliably excluded. The reliability of this measure was $\operatorname{ICC}(2)=0.99$.

\section{Corpus callosum (CC)}

The CC was measured on the sagittal slices, on the midsagittal slice and five slices parasagittally in each direction for a total of eleven slices. Care was taken to exclude the fornix, the septum pellucidum, and the cingulate gyrus. The midsagittal slice was the one on which the cerebral aqueduct becomes continuous with the fourth ventricle. The entire corpus callosum was traced, including the genu, the body, and the splenium. The reliability of this measure is $\operatorname{ICC}(2)=0.95$.

Pons

The pons was measured on the midsagittal slice and two slices parasagittally in each direction for a total of five slices. The entire ovoid contour of the pons was traced with care taken to exclude the medulla and surrounding brainstem. The reliability of this measure is $\operatorname{ICC}(2)=0.94$. 


\section{Statistical analyses}

We analyzed the brain volumetric data with the latent difference model (LDM; McArdle and Nesselroade, 1994). Before the analyses, all regional volumes were adjusted for the volume of the intracranial vault through a regression-ANCOVA approach described in previous publications (Jack et al., 1989; Raz et al., 2004). The LDM model has been applied successfully before to similar data and allows for testing of average sample (mean) change across any two occasions, individual differences (variance) around the sample mean change, and the effects of covariates on individual differences in initial volume and subsequent change. The model takes maximal advantage of bihemispheric measures by defining at each occasion latent ROI volumes uncontaminated by measurement error. The structural relations of the model allow clear differentiation between the baseline volume, change in volume between baseline (Time 1) and the first follow-up (Time 2), change in volume between Time 2 and the second follow-up (Time 3), within a single model. For further detail, see McArdle and Nesselroade (1994) and McArdle (2009) and for applications to volumetric brain data see Raz et al. $(2005,2008)$.

\section{Results}

\section{Descriptive statistics}

Descriptive statistics for ICV-adjusted regional volumes (in $\mathrm{cm}^{3}$ ) summed across hemisphere are displayed in Table 1. In Table 2, we present correlations between volume of each ROI and age at each measurement occasion as well as the standardized differences between the means at baseline and at the first follow-up and between the means at first and second follow-ups (effect sizes; Cohen, 1977). The same table contains the stability coefficients for both intervals. Stability of the measures was very high between Time 1 and Time 2: Pearson correlations range from $r=0.89$ to $r=0.99$, median $r=0.97$, with only one lower than 0.90 , all $p<0.001$. Between Time 2 and Time 3 the stability was lower but still very good for most of the ROIs, with exception of the pons $(r=0.59)$ and the putamen $(r=0.77)$. The range of stability coefficients ranged between $r=0.59$ and $r=0.96$, with a median of $r=0.88$. These coefficients of stability compare favorably to a recently reported set of longitudinal measures (Driscoll et al., 2009). The individual trajectories of change across three measurement occasions over chronological age are presented for each ROI in Figs. 3-6 and a comparison of regional two-occasion changes in Fig. 7.

\section{Latent difference models}

To circumvent the negative consequences of less than perfect reliability of measurement, which are particularly pronounced for the measurement of change (Cronbach and Furby, 1970; cf. Rogosa and Willett, 1985; Baltes et al., 1988), we used latent difference modeling (LDM; McArdle and Nesselroade, 1994; for previous applications of this method to brain volume changes, see Raz et al. (2005, 2008)). LDM handles mean change and individual differences (i.e. variance) in change at the level of latent factors, which are free of measurement error. As in Raz et al. (2005, 2008), ROIs were defined as latent factors representing the variance common to the two hemispheres of a given ROI at each measurement occasion. For example, a latent factor of hippocampal volume at baseline ( time T1) was estimated by the volume of the left and right hippocampi measured at that occasion. The same was done for the two follow-ups (times T2 and T3). This procedure resulted in the specification of three components: (a) hippocampal volume at T1; (b) the change in hippocampal volume from T1 to T2, expressed as the T2 - T1 latent difference score; and (c) the change in hippocampal volume from $\mathrm{T} 2$ to $\mathrm{T} 3$, expressed as the $\mathrm{T} 3-\mathrm{T} 2$ latent difference score. Hence, the method used here extends LDM from the two-occasion to the three-occasion case (cf. McArdle and Nesselroade, 1994; McArdle, 2009).

Within the LDM applied to each ROI, we estimated the mean volume at baseline, the mean change in volume from baseline to Time 2, and the mean change in volume from Time 2 to Time 3. We also estimated the magnitude of individual differences (variance) in baseline volume, in change from baseline to Time 2, and in change from Time 2 to Time 3 around the respective means. Finally, three covariances among the baseline volume, volume change from baseline to Time 2 and change from Time to Time 3 were also estimated. The models fitted the data for every ROI well $\left(\chi^{2}(12)\right.$ ranged from 6.99 to 43.44 with a mean of 16.05 ). The second step involved adding to the LDM, one-at-a-time, time-invariant covariates: age at baseline, education, presence of ApoE\&4 allele, sex, and diagnosis of hypertension at baseline.

\section{Mean change}

The latent model analyses (summarized in Table 3) revealed significant mean shrinkage of the hippocampus, entorhinal cortex, orbital-frontal cortex, and cerebellum over each measurement interval. Additional regions evidenced volume loss only between two occasions. The caudate nucleus and two white matter regions (subcortical prefrontal and corpus callosum) showed no significant loss at the first follow-up but shrunk significantly between the second and the third assessments. For these three ROIs, the difference in rate of change differed significantly among the intervals: difference in $\chi^{2}$ $(1)=4.47,16.89$, and $4.15, p^{\prime} s<0.05$, respectively. In contrast, insula exhibited decline in volume only between baseline and the first follow-up measurement. No changes in the mean volume occurred in the lateral prefrontal and primary visual cortices, the putamen, or the pons. Notably, shrinkage of the hippocampus was greater at the

Table 1

Descriptive statistics for ROI volumes at three measurement occasions.

\begin{tabular}{|c|c|c|c|c|c|c|c|c|c|}
\hline & \multicolumn{3}{|l|}{ Time 1} & \multicolumn{3}{|l|}{ Time 2} & \multicolumn{3}{|l|}{ Time 3} \\
\hline & Mean & SD & $\mathrm{CV}$ & Mean & SD & $\mathrm{CV}$ & Mean & SD & $\mathrm{CV}$ \\
\hline Hippocampus & 6.05 & 0.73 & 0.12 & 5.96 & 0.70 & 0.12 & 5.70 & 0.59 & 0.10 \\
\hline Entorhinal cortex & 2.06 & 0.275 & 0.13 & 1.01 & 0.13 & 0.12 & 1.97 & 0.25 & 0.13 \\
\hline Primary visual cortex & 5.08 & 0.91 & 0.18 & 5.04 & 0.89 & 0.18 & 5.09 & 0.89 & 0.17 \\
\hline Lateral prefrontal cortex & 19.34 & 2.6 & 0.13 & 19.31 & 2.33 & 0.12 & 19.51 & 2.56 & 0.13 \\
\hline Orbital frontal cortex & 9.03 & 1.12 & 0.12 & 8.88 & 1.03 & 0.12 & 8.52 & 0.89 & 0.10 \\
\hline Insula & 7.95 & 0.85 & 0.11 & 7.89 & 0.85 & 0.11 & 7.95 & 0.96 & 0.12 \\
\hline Frontal white & 39.64 & 5.64 & 0.14 & 39.41 & 5.37 & 0.14 & 36.81 & 5.44 & 0.15 \\
\hline Caudate & 7.16 & 0.91 & 0.13 & 7.11 & 0.97 & 0.14 & 6.86 & 0.96 & 0.14 \\
\hline Putamen & 8.70 & 0.82 & 0.09 & 8.63 & 0.81 & 0.09 & 8.41 & 0.85 & 0.10 \\
\hline Cerebellar hemispheres & 109.77 & 12.41 & 0.11 & 108.64 & 12.22 & 0.11 & 106.85 & 11.69 & 0.11 \\
\hline Corpus callosum & 3.47 & 0.47 & 0.14 & 3.49 & 0.45 & 0.13 & 3.44 & 0.37 & 0.11 \\
\hline Ventral pons & 0.91 & 0.11 & 0.13 & 0.91 & 0.10 & 0.11 & 0.94 & 0.12 & 0.13 \\
\hline
\end{tabular}

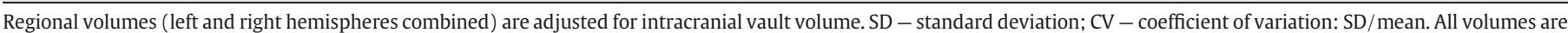
in $\mathrm{cm}^{3}$. 
Table 2

Correlations of regional volumes with calendar age, stability coefficients, and the magnitude of change (effect size) for three measurement occasions.

\begin{tabular}{|c|c|c|c|c|c|c|c|}
\hline ROI & Age at baseline & Age at first follow-up & Age at second follow-up & $r_{12}$ & $r_{23}$ & $d_{12}$ & $d_{23}$ \\
\hline Hippocampus & $-0.45^{* *}$ & $-0.46^{* *}$ & $-0.50^{* *}$ & $0.96^{* *}$ & $0.88^{* *}$ & $0.46^{*}$ & $0.91^{*}$ \\
\hline Entorhinal cortex & -0.03 & 0.01 & -0.06 & $0.97^{* *}$ & $0.83^{* *}$ & $0.60^{*}$ & $0.40^{*}$ \\
\hline Primary visual cortex & -0.13 & -0.07 & 0.01 & $0.99^{* *}$ & $0.96^{* *}$ & 0.20 & 0.24 \\
\hline Lateral prefrontal cortex & $-0.43^{* *}$ & $-0.33^{*}$ & -0.29 & $0.95^{* *}$ & $0.93^{* *}$ & 0.05 & -0.31 \\
\hline Orbital frontal cortex & -0.18 & -0.16 & -0.24 & $0.94^{* *}$ & $0.88^{* *}$ & $0.41^{*}$ & $0.42^{*}$ \\
\hline Insula & -0.17 & -0.14 & -0.00 & $0.98^{* *}$ & $0.79^{* *}$ & $0.41^{*}$ & -0.01 \\
\hline Prefrontal white matter & $-0.36^{*}$ & $-0.39^{*}$ & $-0.41^{*}$ & $0.97^{* *}$ & $0.93^{* *}$ & 0.15 & $0.96^{*}$ \\
\hline Caudate nucleus & -0.20 & -0.23 & $-0.32 \#$ & $0.97^{* *}$ & $0.89^{* *}$ & 0.19 & $0.45^{*}$ \\
\hline Putamen & $-0.46^{* *}$ & $-0.45^{* *}$ & $-0.33 \#$ & $0.96^{* *}$ & $0.77^{* *}$ & 0.24 & 0.25 \\
\hline Cerebellar hemispheres & $-0.31 \#$ & $-0.33^{*}$ & $-0.43^{*}$ & $0.98^{* *}$ & $0.95^{* *}$ & $0.48^{*}$ & $0.56^{*}$ \\
\hline Corpus callosum & -0.13 & -0.07 & 0.06 & $0.91^{* *}$ & $0.82^{* *}$ & 0.12 & $0.45^{*}$ \\
\hline Ventral pons & 0.03 & 0.19 & $0.33 \#$ & $0.89^{* *}$ & $0.59^{* *}$ & 0.11 & -0.18 \\
\hline
\end{tabular}

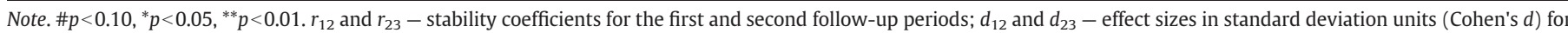

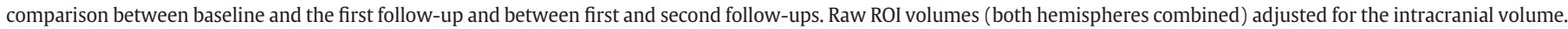

second rather than the first interval: the difference in $\chi^{2}(1)$ was $+8.84, p<0.05$. In other words, the rate of shrinkage of the hippocampus, the caudate, the prefrontal white matter and the corpus callosum accelerated with time. In contrast, the declines in cerebellum, entorhinal, and orbital-frontal volumes did not show reliable deviations from a linear trajectory of decline $(p>0.05)$. The magnitude of mean change varied across the regions (see the effect size comparison in Table 2). The greatest mean 30-month change was observed in the hippocampus and the smallest, not significantly different from zero, in the pons.

\section{Variance of change}

At baseline, all regional volumes exhibited non-zero variance, that is, reliable individual differences. Significant individual differences in the rate of change were observed in LPFC, cerebellum, and all white matter regions in both follow-up intervals. Moreover, in the
Lateral Prefrontal Cortex

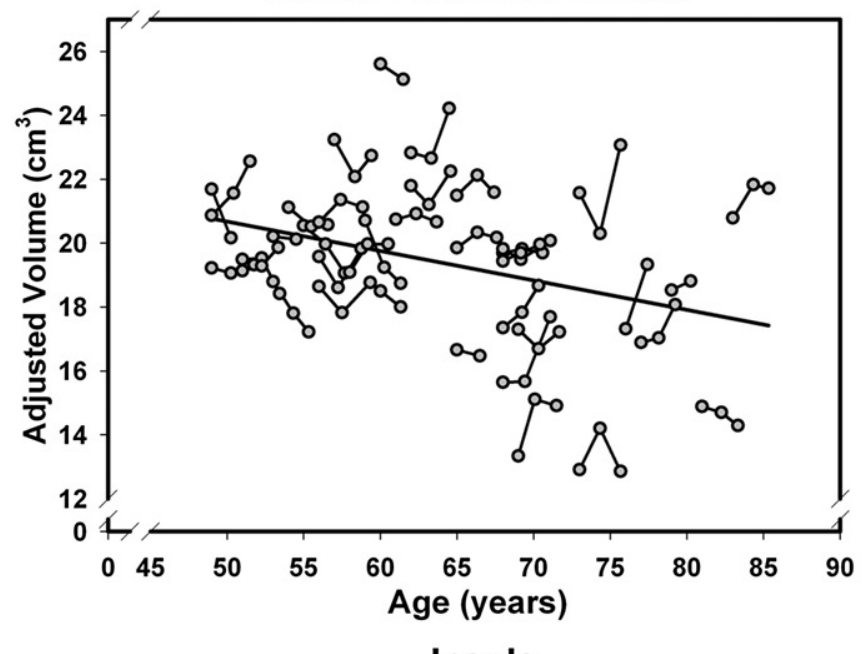

Insula

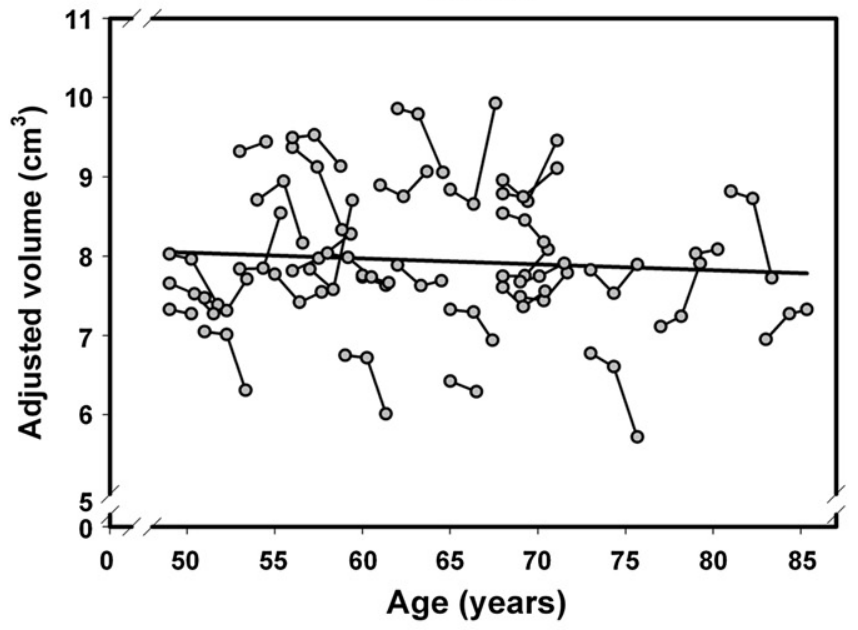

Orbito-frontal Cortex

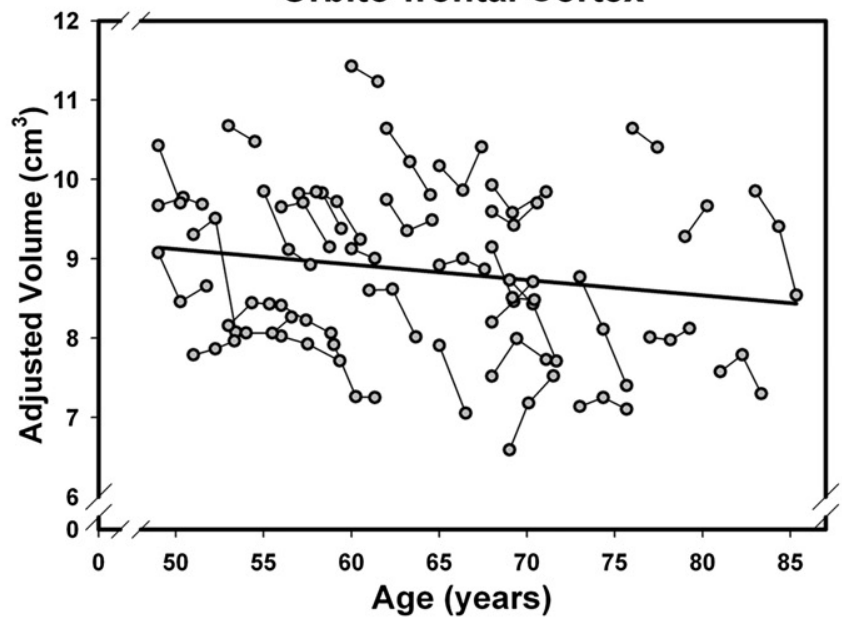

Pericalcarine Cortex

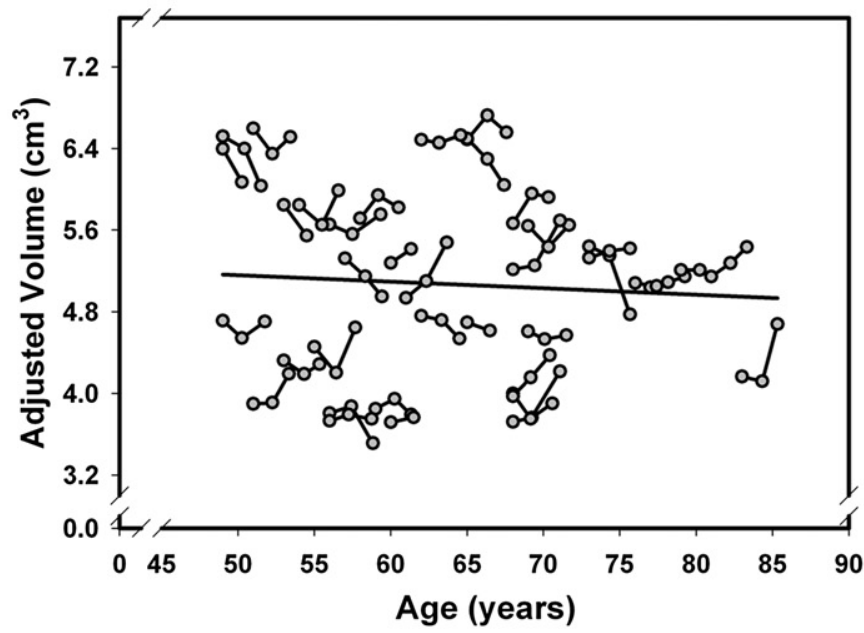

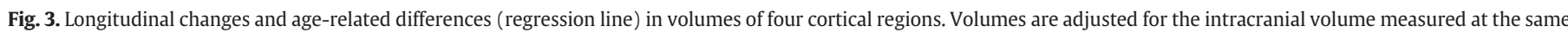
occasion. 

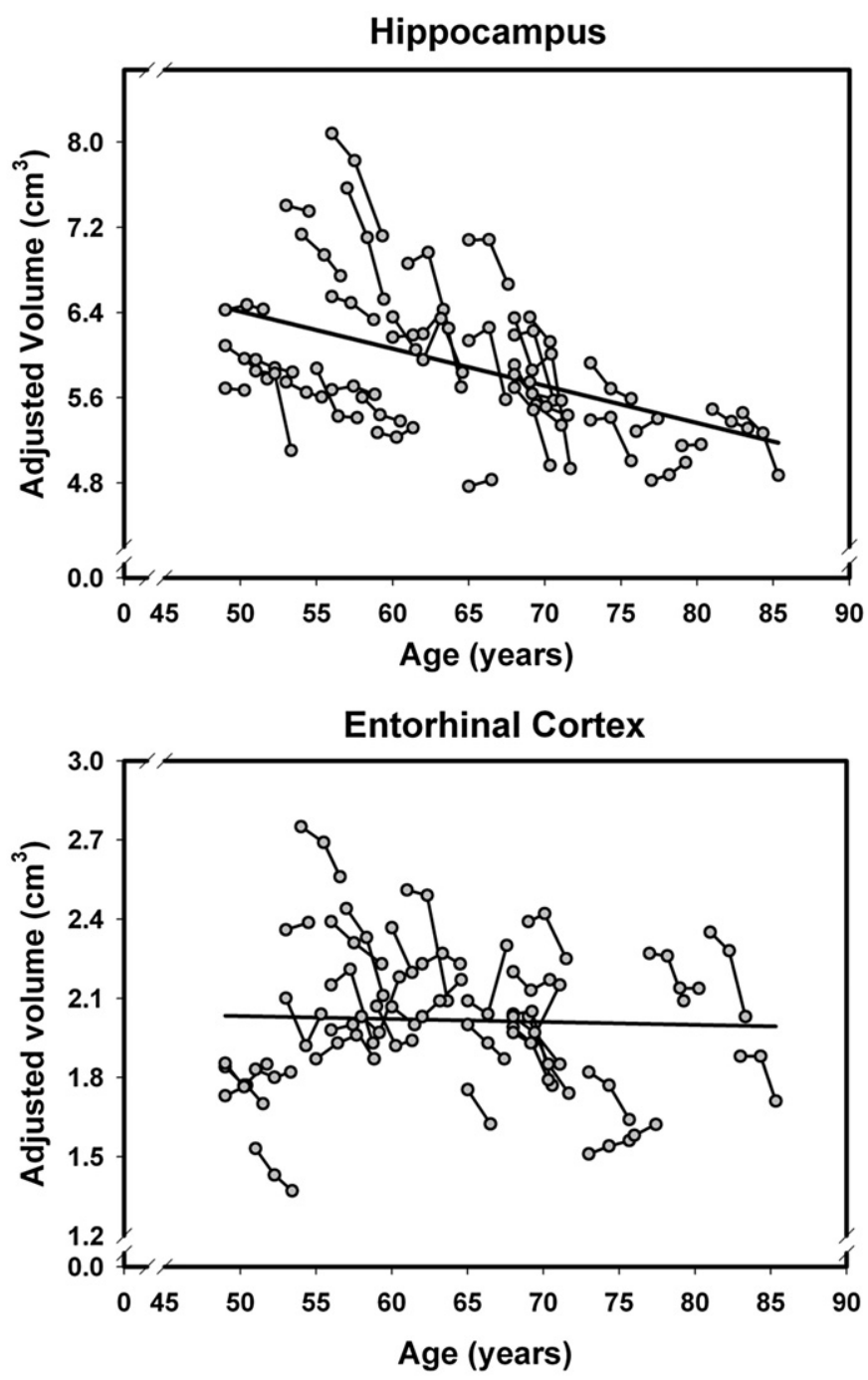

Fig. 4. Longitudinal changes and age-related differences (regression line) in volumes of the medial temporal regions (hippocampus and the entorhinal cortex). Volumes are adjusted for the intracranial volume measured at the same occasion.

cerebellum, corpus callosum, and pons individual differences in shrinkage increased over time (difference in $\chi^{2}$ for $1 \mathrm{df}=13.77$, 5.86 , and $9.18, p^{\prime} s<0.05$, respectively). In five additional regions (HC, $\mathrm{EC}$, insula, caudate and putamen) individual differences in change were absent at the first follow-up assessment but emerged in the course of the second follow-up: difference in $\chi^{2}(1)=9.65,20.33$, $50.21,5.74$, and $26.11, p$ 's $<0.05$, respectively. Two regions, one with a significant mean shrinkage (the orbital-frontal cortex) and the other with no measurable mean change (the primary visual cortex), showed no reliable variance in change across both intervals.

\section{Potential modifiers of brain shrinkage}

The brain reserve hypothesis (Satz, 1993; Stern, 2009) postulates that larger brains may be less vulnerable to progressive pathological processes. Therefore, we examined whether the rate of shrinkage was slower in the participants with larger initial ROI volumes. To that end, we computed the correlations between baseline (T1) and change in the first follow-up interval $(\Delta 12)$, between the baseline (T1) and change in the second follow-up interval $(\Delta 23)$, and between changes in each follow-up interval. Because not all the variances of change were significant, not all correlations among these variables were defined. We found that of the 20 defined correlations, only four were significant: for the hippocampus $\left(r_{\mathrm{T} 1, \Delta 23}=-0.52\right)$, the lateral prefrontal cortex $\left(r_{\mathrm{T} 1, \Delta 12}=-0.50\right)$, the corpus callosum $\left(r_{\mathrm{T} 1, \Delta 23}=-0.38\right)$, and for the pons $\left(r_{\mathrm{T} 1, \Delta 12}=-0.56\right)$. Notably, all significant correlations and 14 out of the remaining 16 non-significant correlations were negative.

To examine the effects of potential modifiers on the course of regional brain aging, we repeated the analyses with five covariates age, sex, education, ApoEs4 status, and diagnosis of hypertension, introduced one-at-a-time. We observed no association between age and shrinkage rate in any of the examined regions. The only significant effect of sex was observed in the pons: women showed significant shrinkage between Time 2 and Time 3 whereas men did not. The only difference between normotensive participants and their hypertensive peers was in the corpus callosum shrinkage rate between the first and the second follow-up measurements: persons with hypertension evidenced steeper declines. To assess the potentially modifying effect of a genetic risk factor, ApoE\&4 allele, we contrasted 10 ApoE\&4 carriers with 26 par-

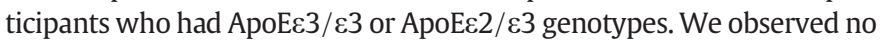
effect of ApoE\&4 allele on shrinkage rate in any of the measured regions except for a modest effect $(-0.11$, unstandardized) on the change between Times 2 and 3 in the pons, indicating some shrinkage in carriers of ApoE\&4 allele.

A variant of the brain reserve hypothesis states that education may be neuroprotective (Coffey et al, 1999). In other words, in the aging process, the brains of persons with high levels of formal education may fare better than those of their less educated peers. In testing that hypothesis, we found no significant correlations between regional rates of change and number of years of formal education at baseline.

Because the interval between the first and the second follow-up assessment varied among the participants, we re-evaluated all models with delay between the two follow-ups as a covariate. As there were no significant changes in the effects or model fit, the interval duration was not included in the models reported here.

\section{Discussion}

The main finding in this study is that brains of healthy individuals undergo significant shrinkage in a relatively short period. However, our findings also establish significant individual variability in the rates of decline. Potential modifiers of brain aging that were suggested by the previous studies, i.e., hypertension, sex, or ApoEc4 allele explain some of the observed variability in change but only in two white matter regions.

Given the dearth of longitudinal studies of healthy brain aging, it is important that the current study replicates many of the previous findings. We observed shrinkage of the orbital-frontal cortex, the cerebellum, the hippocampus, the subcortical prefrontal white matter and the caudate, and relative stability of the primary visual cortex (Raz et al., 2005; Driscoll et al., 2009; Fjell et al., 2009b). Stability of the pons volume was confirmed for the whole sample but shrinkage was noted in women and carriers of ApoE\&4. Moreover, we replicated the nonlinear (accelerated) shrinkage of the hippocampus (Raz et al., 2005; Fjell et al., 2009b). However, some of the previous findings were not replicated. Most notably, the lack of mean change in the lateral prefrontal cortex, a region that evidenced age-related declines in several samples (Pfefferbaum et al., 1998; Resnick et al., 2003; Raz et al., 2005; Driscoll et al., 2009; Fjell et al., 2009b) and the putamen, in which age-related shrinkage has been reported (Raz et al., 2003a; Fjell et al., 2009b). Although in this investigation, the intervals between measurements were substantially shorter than in most of the extant studies, it is unclear whether that was the reason for lack of observed change (cf. Fjell et al., 2009b). We observed reliable shrinkage of the entorhinal cortex that seems to exceed the magnitude of change observed in our previous study (Raz et al., 2005). It is important to note, that in that sample and several others (e.g. Du et al., 2006; Fjell et al., 2009b) age-related change in EC was apparent towards the seventh decade of age. It is plausible that the discrepancies stem from the sample characteristics or the time window used in this study. At least as far as the measured demographic and health characteristics are 


\section{Caudate Nucleus}

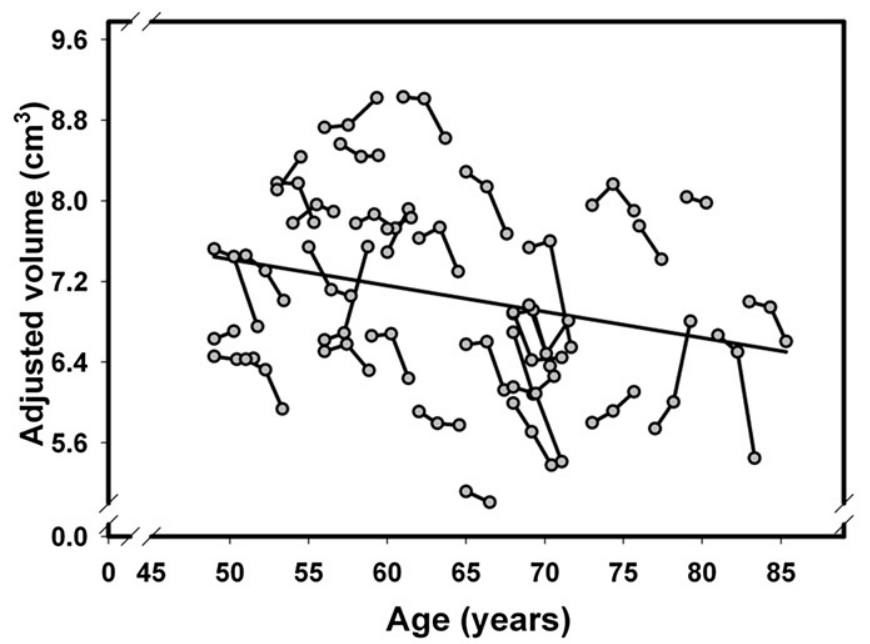

Putamen

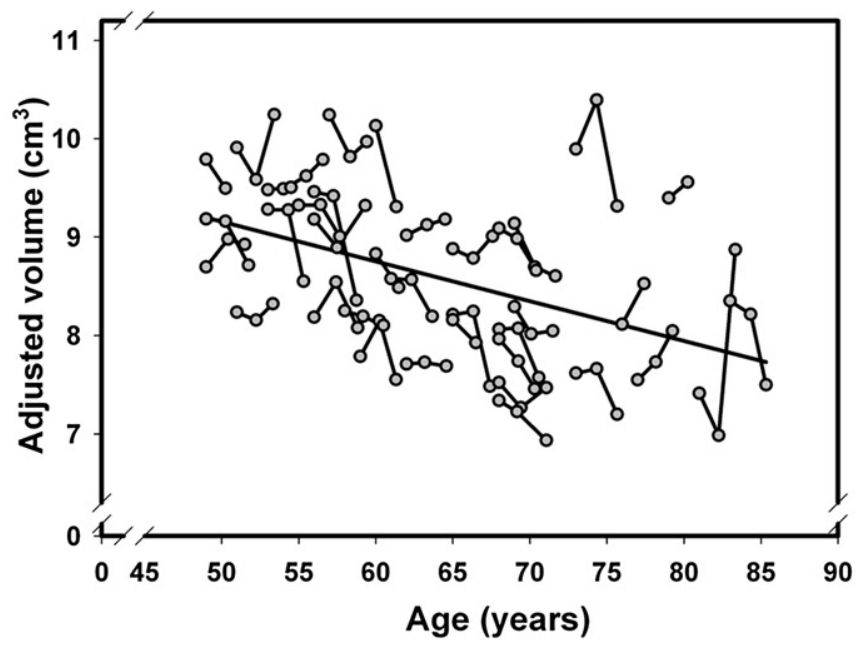

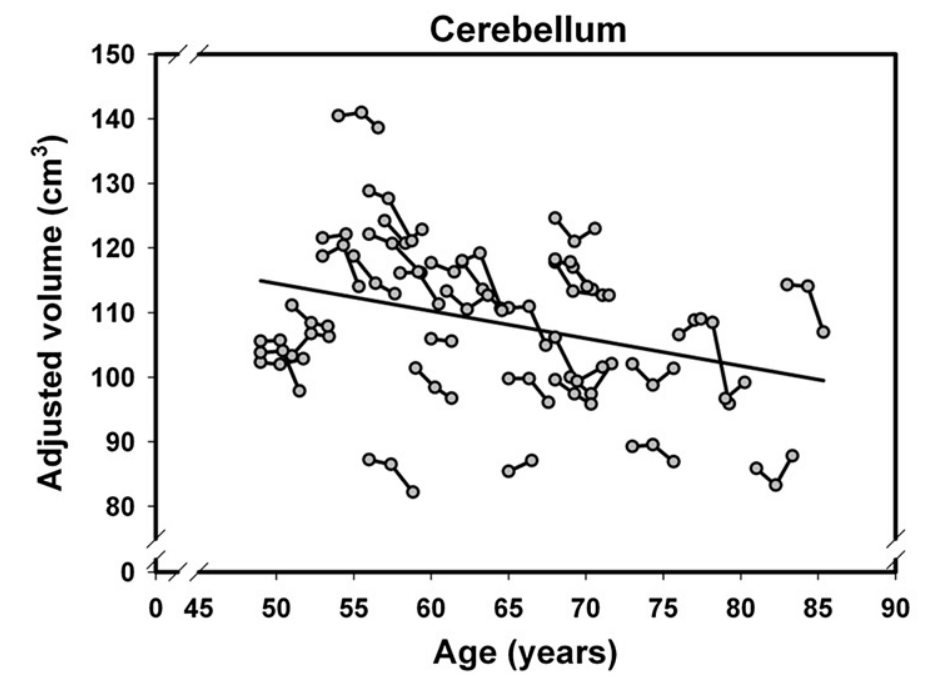

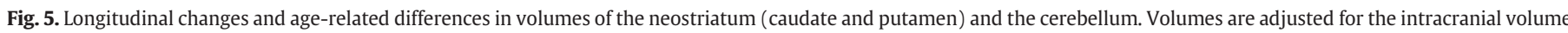
measured at the same occasion.

concerned, the former is unlikely. Although the current sample originated from a different geographic area, it was demographically very similar to the previous one. Thus, in all likelihood, the observed changes are due to increased proportion of older participants. Furthermore, although the participants in the current study maintained a normal cognitive status throughout the study, one has to keep in mind that entorhinal shrinkage has been linked to cognitive declines (de Toledo-Morrell et al., 2000) and lower cognitive performance in normal elderly (Rodrigue and Raz, 2004; Raz et al., 2008). It is possible, therefore, that the observed change in entorhinal volume signals impending cognitive decline that can be established only in the future follow-ups.

Life-span brain development is regionally heterochronic. While white matter volume increases steadily with maturation into the middle adulthood, the volume of the gray matter structures such as the caudate, the cerebellum, and the neocortex peak at early adolescence and steadies or declines thereafter (Lenroot and Giedd, 2006; Tiemeier et al., 2009). Most of the current longitudinal findings, while establishing heterochronicity of change at the group level do little to address individual variations in trajectories of aging and development. Heterogeneity of individual paths may be such that two- and three-wave longitudinal studies are insufficiently sensitive to highly variable and possibly non-monotonic trajectories of change. The state of brain structure at a given moment is a product of a dynamic equilibrium of multiple biochemical, metabolic, and mechanical forces and events.
For any individual and any brain region, the direction of the moment-tomoment structural change may vary, thus creating a time series with a clear trend but with substantial quasi-periodic variability. Thus, the question of specific timing of the adult changes in the brain volumes may require more fine-grained measurements that would faithfully sample intra-individual fluctuations in the timing of regional shrinkage.

In this context, the important finding in this study is the demonstration of significant and widespread individual differences in change. It is worth noting that in this study, the heterogeneity of change trajectories was especially pervasive in the white matter compartment that undergoes significant volumetric and structural transformations throughout the life-span (Bartzokis et al., 2004; Kennedy and Raz, 2009; Madden et al., 2009). Substantial individual variability in short-term aging trajectories was noted even in the regions that evidenced no mean change, a finding that was made possible by application of the LDM methods. Notably, heterogeneity emerged and intensified over time. Even in the regions that showed uniform change between baseline and the first follow-up assessment, significant individual differences appear during the second follow-up interval. Such a pattern suggests the influence of age-related factors, whose modifying influence becomes apparent with time. What those accelerators of aging are remains unclear; examining the "usual suspects," such as genetic and vascular risk, education, and sex explained little of that variability. Nonetheless, in one region (corpus callosum), we replicated exacerbating effect of hypertension on age- 


\section{Pre-Frontal White Matter}

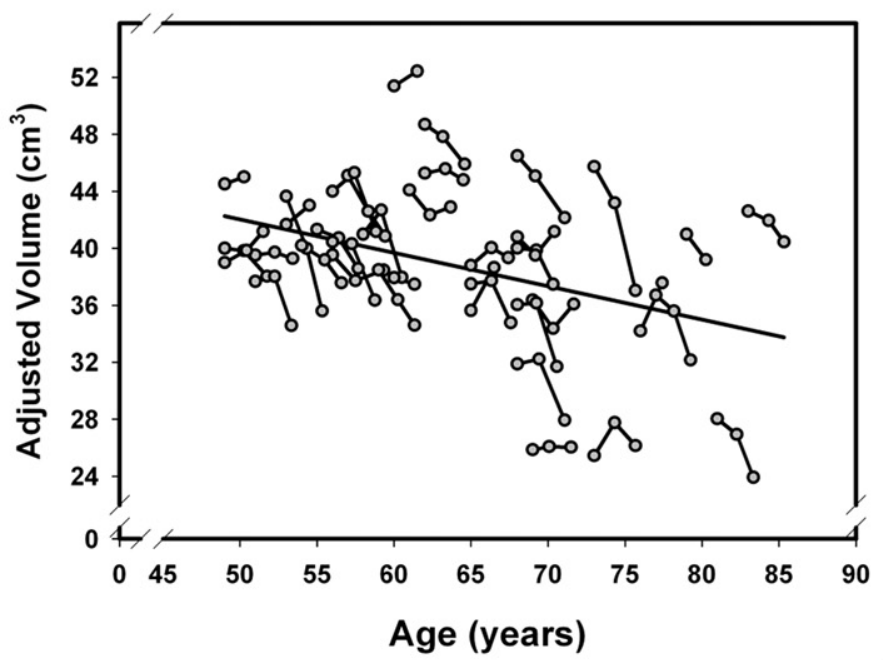

Corpus Callosum

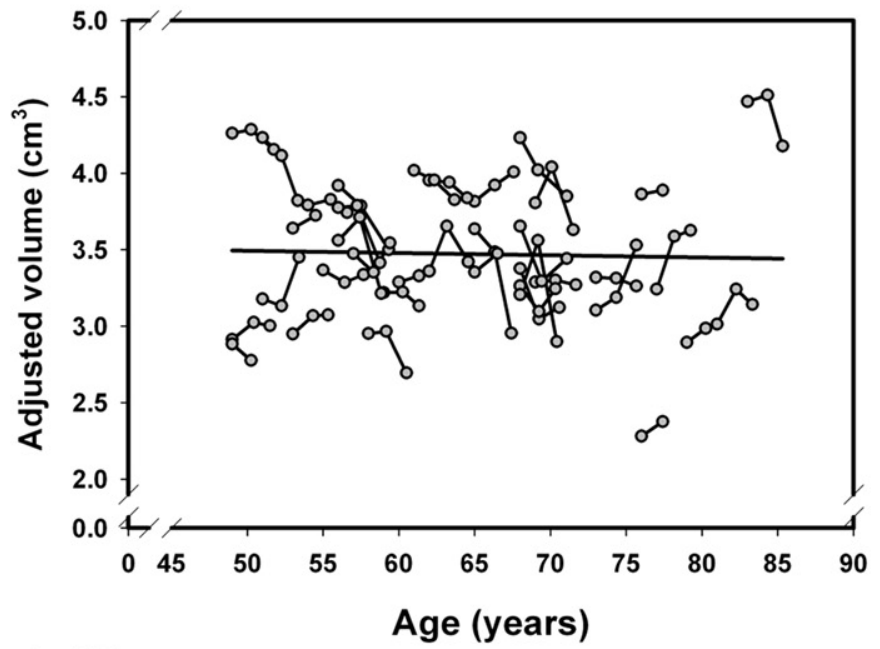

Ventral Pons

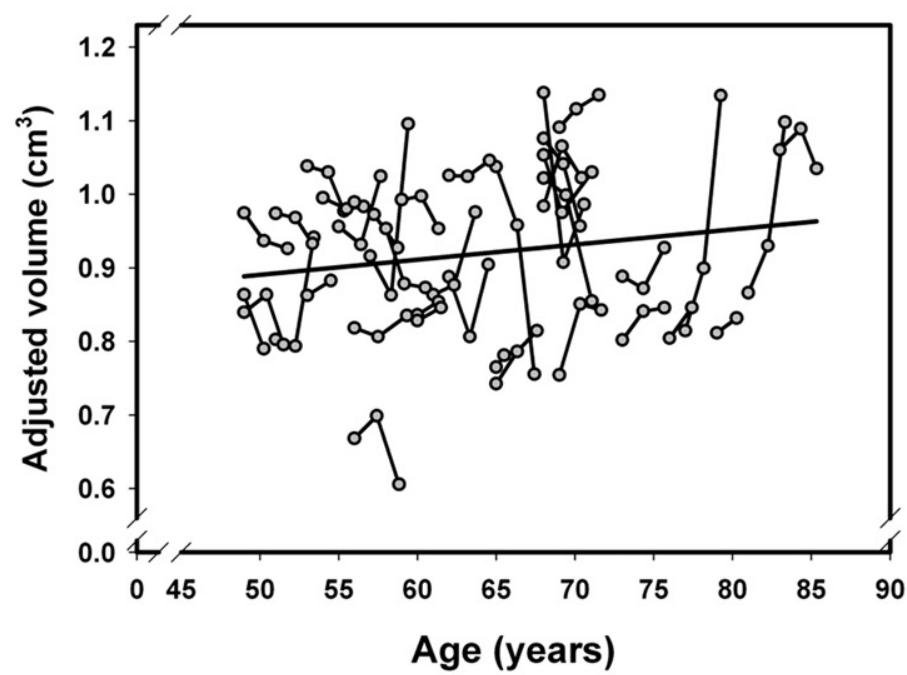

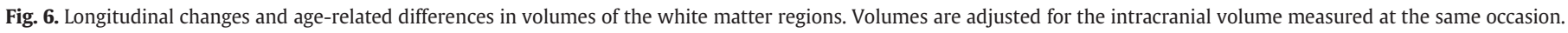

related shrinkage (Harris et al., 2008). Although in a relatively small sample, the lack of finding can be attributed in part to the low statistical power (Hertzog et al., 2006), it also indicates that none of the candidate modifiers produced a dramatic effect on the rate of decline, and that further attention should be devoted to identifying the mechanisms of individual variability of regional brain aging.

Although testing the brain reserve hypothesis was not a major goal of this study, the results provided are pertinent to both passive and active reserve hypotheses. The former predicts that larger brains would show slower decline and greater resistance to cumulative damage (Satz, 1993), whereas the latter proposed that education would be a marker of neuroprotection (Coffey et al., 1999). Although the current study does not address issue of reserve in its complexity (for a concise review see Stern (2009)), we found no evidence in support of cognitive or brain reserve hypotheses. Neither baseline volume nor educational attainment had any effect on regional brain shrinkage. If anything, the opposite was true: larger regions evidenced steeper absolute declines. The apparent lack of protection conveyed by mere size is a replication of our previous finding (Raz et al., 2005), whereas the negative results for education agree with a previous report (Christensen et al., 2007). In part, the negative findings may reflect the particular characteristics of this study: better than average health status of the participants, with only less than $25 \%$ of the respondents accepted into the study, high educational attainment at almost a full college level, and short duration of the follow-up.

The ApoE\&4 allele, a known risk factor for AD, showed little influence in this sample of healthy adults. An upward selection bias in participant recruitment may account for lack of this effect. The ApoE\&4 carriers who were free of dementia and cardiovascular disease at the inception of the study and who managed to remain healthy throughout the follow-up could have had other genetic or physiological factors offsetting the negative effect of that genetic variant. Interestingly, the only region on which ApoE\&4 exerted negative influence was the pons, usually deemed resistant to age-related changes (Raz et al., 2005). It is also notable that the pons was the only region in which individual variability of volume change was significantly affected by sex. These findings, though potentially random, are nonetheless intriguing and worthy of further examination.

In sum, this study demonstrated that differential shrinkage of brain parenchyma might occur in healthy middle-aged and older adults within a relatively short period and with no apparent pathological influences. The pace and the magnitude of those changes vary substantially among the individuals, but it is apparent that several established modifiers of brain aging could not explain individual variability in its course. It is clear, 
Changes in Regional Cortical Volumes
Baseline to First Follow-up

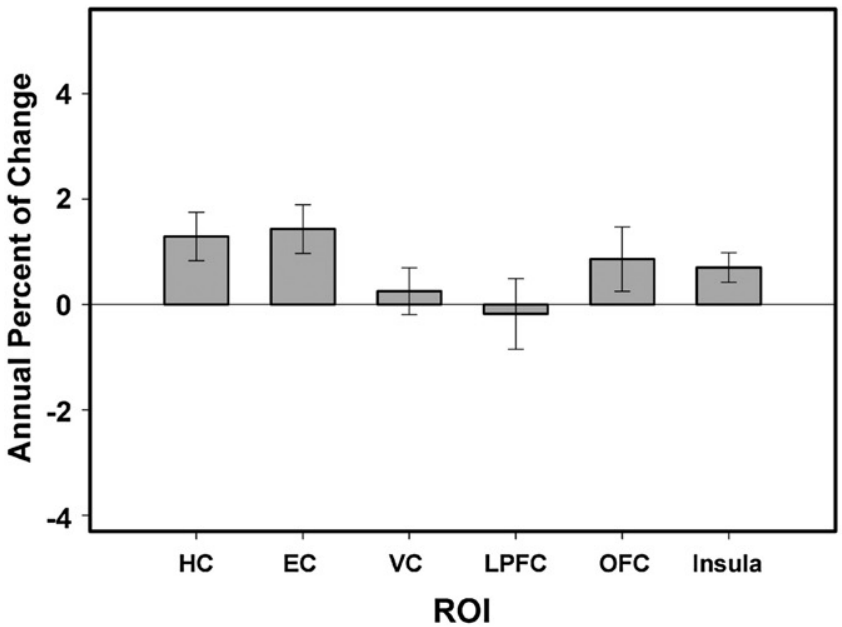

First to Second Follow-up

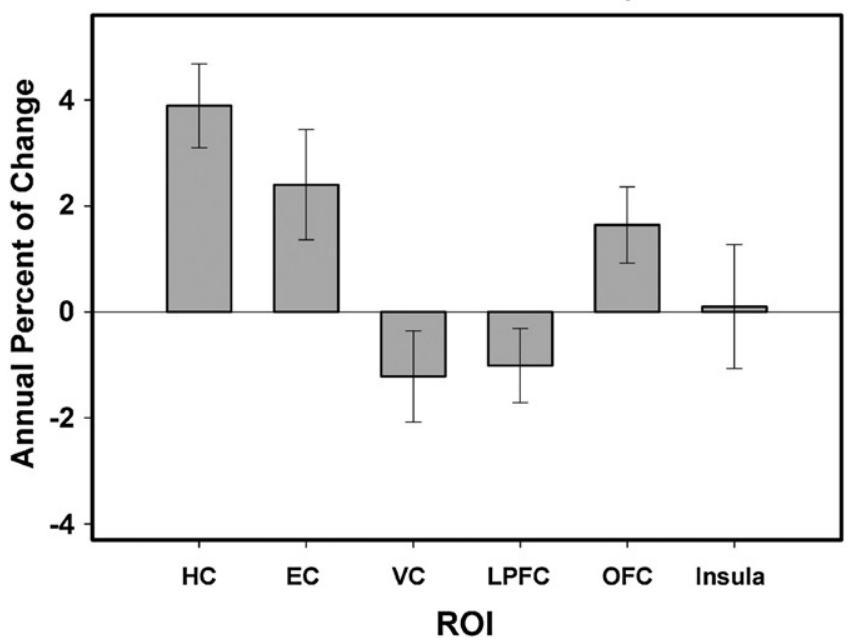

Change in Volumes of Basal Ganglia and Metencephalon From Baseline to First Follow-Up

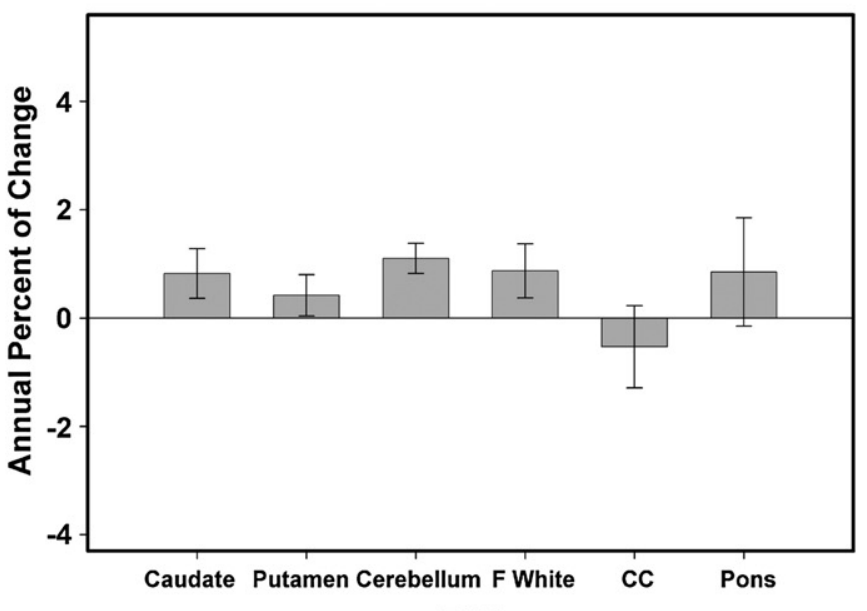

ROI

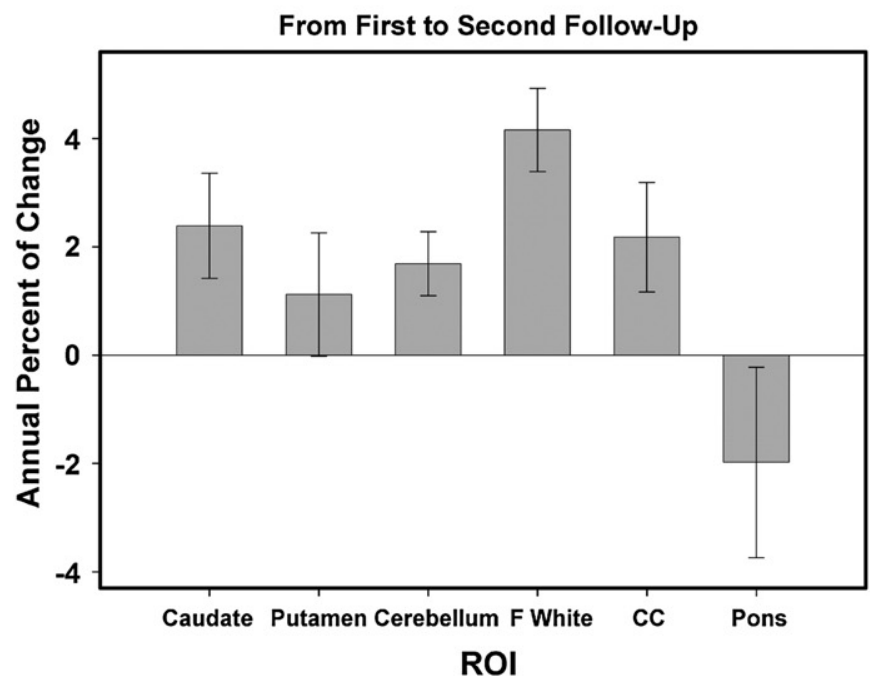

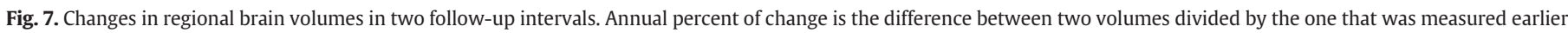
between the two and by the length of the interval between the measurements. The bars are standard errors of the means.

Table 3

Longitudinal change in regional cerebral volumes: a summary of the latent difference models.

\begin{tabular}{|c|c|c|c|c|c|c|c|c|}
\hline \multirow[t]{2}{*}{ ROI } & \multicolumn{4}{|l|}{ Mean } & \multicolumn{4}{|c|}{ Variance } \\
\hline & $\mathrm{T} 1$ & $\Delta 12$ & $\Delta 23$ & $\Delta 12=\Delta 23$ & $\mathrm{~T} 1$ & $\Delta 12$ & $\Delta 23$ & $\Delta 12=\Delta 23$ \\
\hline \multicolumn{9}{|c|}{ Cortical regions and subcortical nuclei } \\
\hline $\mathrm{HC}$ & $3.06^{*}$ & $-0.04^{*}$ & $-0.16^{*}$ & * & $0.11^{*}$ & 0.00 & $0.02 *$ & * \\
\hline EC & $1.28^{*}$ & $-0.02^{*}$ & $-0.03^{*}$ & ns & $0.02^{*}$ & 0.00 & $0.01^{*}$ & * \\
\hline LPFC & $8.98^{*}$ & -0.02 & 0.15 & ns & $0.14^{*}$ & $0.12^{*}$ & $0.15^{*}$ & ns \\
\hline OFC & $4.32^{*}$ & $-0.09^{*}$ & $-0.10^{*}$ & ns & $0.26^{*}$ & 0.01 & 0.02 & ns \\
\hline VC & $2.52^{*}$ & -0.02 & 0.03 & ns & $0.15^{*}$ & 0.00 & 0.01 & ns \\
\hline In & $3.95^{*}$ & $-0.03^{*}$ & -0.01 & ns & $0.16^{*}$ & 0.00 & $0.08^{*}$ & $*$ \\
\hline $\mathrm{Cd}$ & $3.72^{*}$ & -0.02 & $-0.12^{*}$ & * & $0.20^{*}$ & 0.00 & $0.02^{*}$ & * \\
\hline $\mathrm{Pt}$ & $3.90^{*}$ & -0.02 & -0.09 & ns & $0.13^{*}$ & 0.00 & $0.06^{*}$ & * \\
\hline $\mathrm{CbH}$ & $57.50^{*}$ & $-0.58^{*}$ & $-1.44^{*}$ & ns & $39.01^{*}$ & $0.86^{*}$ & $3.61^{*}$ & $*$ \\
\hline \multicolumn{9}{|c|}{ White matter } \\
\hline PFw & $20.38^{*}$ & -0.07 & $-1.14^{*}$ & $*$ & $7.34^{*}$ & $0.46^{*}$ & $0.75^{*}$ & ns \\
\hline CC & $3.48^{*}$ & 0.02 & $-0.10^{*}$ & $*$ & $0.21^{*}$ & $0.03^{*}$ & $0.06^{*}$ & $*$ \\
\hline Pons & $0.91^{*}$ & -0.01 & 0.02 & ns & $0.01^{*}$ & $0.003^{*}$ & $0.01^{*}$ & * \\
\hline
\end{tabular}

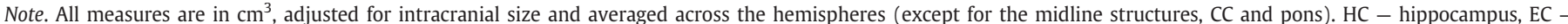

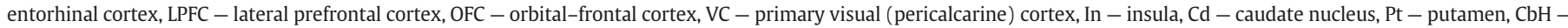

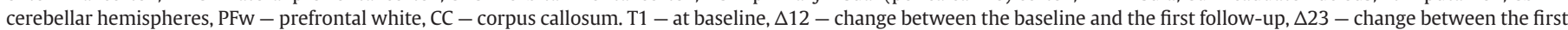
and the second follow-ups. $\Delta 12=\Delta 23$ refers to the significance of the tests of equality of mean change and variance change between the two adjacent follow-ups. ${ }^{*} p<0.05$. 
however, that regional volumetric analysis is a valid and sensitive tool for assessing brain change, and further search for modifiers that speed or retard brain aging is in order.

\section{Acknowledgment}

This project was supported by grants R37 AG-011230 and R03 AG024630 from the National Institute on Aging, USA.

\section{References}

Baltes, P.B., Reese, H.W., Nesselroade, J.R., 1988. Life-span Developmental Psychology: an Introduction to Research Methods. Lawrence Erlbaum Associates, Hillsdale, NJ. (reprint of 1977 ed.).

Barnes, J., Bartlett, J.W., van de Pol, L.A., Loy, C.T., Scahill, R.I., Frost, C., Thompson, P., Fox, N.C. 2009. A meta-analysis of hippocampal atrophy rates in Alzheimer's disease. Neurobiol. Aging 30, 1711-1723 (Electronic publication ahead of print, 2008 Mar 17).

Bartzokis, G., Lu, P.H., Mintz, J., 2004. Quantifying age-related myelin breakdown with MRI: novel therapeutic targets for preventing cognitive decline and Alzheimer's disease. J Alzheimers Dis 6 (6 Suppl), S53-S59.

Christensen, H., Anstey, K.J., Parslow, R.A., Maller, J., Mackinnon, A., Sachdev, P., 2007. The brain reserve hypothesis, brain atrophy and aging. Gerontology 53, 82-95 Electronic publication ahead of print 2006 Oct 20

Coffey, C.E., Saxton, J.A., Ratcliff, G., Bryan, R.N., Lucke, J.F., 1999. Relation of education to brain size in normal aging: implications for the reserve hypothesis. Neurology 53 , 189-196.

Cohen, J., 1977. Statistical power analysis for the behavioral sciences (revised edition) Academic Press, New York.

Cronbach, L.J., Furby, L., 1970. How we should measure "change" - or should we? Psychol. Bull. 74, 68-80.

de Toledo-Morrell, L., Goncharova, I., Dickerson, B., Wilson, R.S., Bennett, D.A., 2000 From healthy aging to early Alzheimer's disease: in vivo detection of entorhinal cortex atrophy. Ann. N. Y. Acad. Sci. 911, 240-253.

Driscoll, I., Davatzikos, C., An, Y., Wu, X., Shen, D., Kraut, M., Resnick, S.M., 2009. Longitudinal pattern of regional brain volume change differentiates normal aging from MCI. Neurology 72, 1906-1913.

Du, A.T., Schuff, N., Chao, L.L., Kornak, J., Jagust, W.J., Kramer, J.H., Reed, B.R., Miller, B.L Norman, D., Chui, H.C., Weiner, M.W., 2006. Age effects on atrophy rates of entorhinal cortex and hippocampus. Neurobiol. Aging 7, 733-740 Electronic publication ahead of print 2005 Jun 14

Eritaia, J., Wood, S.J., Stuart, G.W., Bridle, N., Dudgeon, P., Maruff, P., Velakoulis, D., Pantelis, C., 2000. An optimized method for estimating intracranial volume from magnetic resonance images. Magn. Reson. Med. 44, 973-977.

Fjell, A.M., Walhovd, K.B., Fennema-Notestine, C., McEvoy, L.K., Hagler, D.J., Holland, D. Brewer, J.B., Dale, A.M., 2009a. One-year brain atrophy evident in healthy aging. J. Neurosci. 29, 15223-15231.

Fjell AM, Westlye LT, Amlien I, Espeseth T, Reinvang I, Raz N, Agartz I, Salat DH, Greve DN, Fischl B, Dale AM, Walhovd KB. 2009a. High consistency of regional cortica thinning in aging across multiple samples. Cerebral Cortex 19, 2001-2012. Epub 2009 Jan 15.

Folstein, M., Folstein, S., McHugh, P., 1975. "Mini-Mental State”: a practical method for grading the cognitive state of patients for the clinician. Journal of Psychiatry Research 12, 189-198.

Harris, P., Alcantara, D.A, Amenta, N., Lopez, O.L, Eiríksdóttir, G., Sigurdsson, S, Gudnason, V., Madsen, S., Thompson, P.M., Launer, L.J., Carmichael, O.T., 2008. Localized measures of callosal atrophy are associated with late-life hypertension: AGES-Reykjavik Study. Neuroimage 43, 489-496 Electronic publication ahead of print 2008 Jul 18.

Hertzog, C., Lindenberger, U., Ghisletta, P., von Oertzen, T., 2006. On the power of multivariate latent growth curve models to detect correlated change. Psychologica Methods 11, 244-252.

Insausti, R., Juottonen, K., Soininen, H., Insausti, A.M., Partanen, K., Vainio, P., Laakso, M.P., Pitkänen, A., 1998. MR volumetric analysis of the human entorhinal, perirhinal, and temporopolar cortices. AJNR Am. J. Neuroradiol. 19, 659-671.

Jack Jr, C.R., Twomey, C.K., Zinsmeister, A.R., Sharbrough, F.W., Petersen, R.C., Cascino, G.D., 1989. Anterior temporal lobes and hippocampal formations: normative volumetric measurements from MR images in young adults. Radiology 172, 549-554.

Jernigan, T.L. Archibald, S.L., Fenema-Notestine, C. Gamst, A.C., Stout, J.C., Bonner, J. Hesselink, J.R., 2001. Effects of age on tissues and regions of the cerebrum and cerebellum. Neurobioloy of Aging 22, 581-594

Joint National Committee on Prevention, Detection, Evaluation, and Treatment of High Blood Pressure, 1997. The sixth report of the Joint National Committee on Prevention, Detection, Evaluation, and Treatment of High Blood Pressure. Arch. Intern. Med. 157, 2413-2446.

Kennedy, K.M., Raz, N., 2009. Pattern of normal age-related regional differences in white matter microstructure is modified by vascular risk. Brain Res. 1297, 41-56.

Lenroot, R.K., Giedd, J.N., 2006. Brain development in children and adolescents: insights from anatomical magnetic resonance imaging. Neurosci. Biobehav. Rev. 30 718-729 Electronic publication ahead of print 2006 Aug 2.
Madden, D.J., Bennett, I.J., Song, A.W., 2009. Cerebral white matter integrity and cognitive aging: contributions from diffusion tensor imaging. Neuropsychol. Rev. 19, 415-435 (Electronic publication ahead of print, 2009 Aug 25).

McArdle, J.J., 2009. Latent variable modeling of differences and changes with longitudinal data. Annu. Rev. Psychol. 60, 577-605.

McArdle, J.J., Nesselroade, J.R., 1994. Using multivariate data to structure developmental change. In: Cohen, S.H., Reese, H.W. (Eds.), Life-span Developmental Psychology: Methodological Contributions. Lawrence Erlbaum Associates, Hillsdale, NJ, pp. 223-267.

McArdle, J.J., Nesselroade, J.R., 2003. Growth curve analysis in contemporary psychological research. In: Schinka, J., Velicer, W. (Eds.), Comprehensive Handbook of Psychology: Research Methods in Psychology, 2. Wiley, New York, N.Y., pp. 447-480.

McArdle, J.J., Hamagami, F., Jones, K., Jolesz, F., Kikinis, R., Spiro, A., et al., 2004. Structural modeling of dynamic changes in memory and brain structure using longitudinal data from the Normative Aging Study. Journals of Gerontology: Psychological Sciences 59B, P294-P304.

Moffat, S.D., Szekely, C.A., Zonderman, A.B., Kabani, N.J., Resnick, S.M., 2000. Longitudinal change in hippocampal volume as a function of apolipoprotein $\mathrm{E}$ genotype. Neurology 55, 134-136.

Oldfield, R.C., 1971. The assessment and analysis of handedness. Neuropsychologia 9, 97-113.

Pfefferbaum, A., Sullivan, E.V., Rosenbloom, M.J., Mathalon, D.H., Lim, K.O., 1998. A controlled study of cortical gray matter and ventricular changes in alcoholic men over a 5-year interval. Arch. Gen. Psychiatry 55, 905-912.

Radloff, L.S., 1977. The CES-D scale: a self-report depression scale for research in the general population. Applied Psychological Measurement 1, 385-401.

Raz, N., Kennedy, K.M., 2009. A systems approach to age-related change: neuroanatomic changes, their modifiers, and cognitive correlates. In: Jagust, W., D'Esposito., M. (Eds.), Imaging the Aging Brain. Oxford University Press, New York, NY, pp. $43-70$

Raz, N., Gunning, F.M., Head, D., Dupuis, J.H., McQuain, J.M., Briggs, S.D., Thornton, A.E., Loken, W.J., Acker, J.D., 1997. Selective aging of human cerebral cortex observed in vivo: differential vulnerability of the prefrontal gray matter. Cereb. Cortex 7, 268-282.

Raz, N., Rodrigue, K.M., Kennedy, K.M., Head, D., Gunning-Dixon, F.M., Acker, J.D., 2003a. Differential aging of the human striatum: longitudinal evidence. American Journal of Neuroradiology 24, 1849-1856.

Raz, N., Rodrigue, K.M., Kennedy, K.M., Dahle, C., Head, D., Acker, J.D., 2003b. Differential age-related changes in the regional metencephalic volumes in humans: a five-year follow-up. Neurosci. Lett. 349, 163-166.

Raz, N., Gunning-Dixon, F., Head, D., Williamson, A., Rodrigue, K., Acker, J.D., 2004. Aging, sexual dimorphism, and hemispheric asymmetry of the cerebral cortex: replicability of regional differences in volume. Neurobiol. Aging 25, 377-396.

Raz, N., Lindenberger, U., Rodrigue, K.M., Kennedy, K.M., Head, D., Williamson, A., Dahle, C., Gerstorf, D., Acker, J.D., 2005. Regional brain changes in aging healthy adults: general trends, individual differences, and modifiers. Cereb. Cortex 15, 1676-1689.

Raz, N., Rodrigue, K.M., Kennedy, K.M., Acker, J.D., 2007. Vascular health and longitudinal changes in brain and cognition in middle-aged and older adults. Neuropsychology 21, 149-157.

Raz, N., Lindenberger, U., Ghisletta, P., Rodrigue, K.M., Kennedy, K.M., Acker, J.D., 2008. Neuroanatomical correlates of fluid intelligence in healthy adults and persons with vascular risk factors. Cereb. Cortex 718-726 Electronic publication ahead of print.

Raz, N., Rodrigue, K.M., Kennedy, K.M., Land, S., 2009. Genetic and vascular modifiers of age-sensitive cognitive skills: effects of COMT, BDNF, ApoE and hypertension. Neuropsychology. 23, 105-116.

Resnick, S.M., Pham, D.L., Kraut, M.A., Zonderman, A.B., Davatzikos, C., 2003. Longitudinal magnetic resonance imaging studies of older adults: a shrinking brain. J. Neurosci. 23, 3295-3301.

Rodrigue, K.M., Raz, N., 2004. Shrinkage of the entorhinal cortex over five years predicts memory performance in healthy adults. J. Neurosci. 24, 956-963.

Rogosa, D.R., Willett, J.B., 1985. Understanding correlates of change by modeling individual differences in growth. Psychometrika 50, 203-228.

Rusinek, H., De Santi, S., Frid, D., Tsui, W.H., Tarshish, C.Y., Convit, A., de Leon, M.J., 2003. Regional brain atrophy rate predicts future cognitive decline: 6-year longitudinal MR imaging study of normal aging. Radiology 229, 691-696.

Satz, P., 1993. Brain reserve capacity on symptom onset after brain injury: a formulation and review of evidence for threshold theory. Neuropsychology 7, 273-295.

Scahill, R.I., Frost, C., Jenkins, R., Whitwell, J.L., Rossor, M.N., Fox, N.C., 2003. A longitudinal study of brain volume changes in normal aging using serial registered magnetic resonance imaging. Arch. Neurol. 60, 989-994.

Shrout, P.E., Fleiss, J.L., 1979. Intraclass correlations: uses in assessing raters reliability. Psychol. Bull. 86, 420-428.

Stern, Y., 2009. Cognitive reserve. Neuropsychologia. 47, 2015-2028 Electronic publication ahead of print 2009 Mar 13.

Stoub, T.R., Rogalski, E.J., Leurgans, S., Bennett, D.A., Detoledo-Morrell, L., 2008. Rate of entorhinal and hippocampal atrophy in incipient and mild AD: relation to memory function. Neurobiol. Aging (2008 Sep 20, Electronic publication ahead of print).

Tiemeier, H., Lenroot, R.K., Greenstein, D.K., Tran, L., Pierson, R., Giedd, J.N., 2009. Cerebellum development during childhood and adolescence: a longitudinal morphometric MRI study. NeuroImage Electronic publication ahead of print. 